\title{
Mano y rostro. La filosofía de la expresión en Leonardo da Vinci y Marsilio Ficino*
}

\section{Gianluca Cuozzo \\ Università di Torino, Italia}

Recibido el 20 de junio de 2019. Aceptado el o6 de agosto de 2019.

\begin{abstract}
Resumen
El presente artículo se centra en Marsilio Ficino y Leonardo da Vinci. Ambos autores describen la producción humana de artefactos como principios transformadores que afectan el mundo a partir de un punto de vista tanto técnico como estético. En este sentido, manus y risus se convierten en bastiones de una antropología típicamente renacentista, según la cual el homo faber se eleva a ese estado divino que juega un importante papel en la filosofía de Nicolás de Cusa; mientras la mano humana es el principio transformador activo, la sonrisa es el signo de un trabajo cumplido. En suma, estos dos aspectos revelan un proceso de espiritualización de la realidad, i.e., la perfecta interpenetración de la belleza con la virtud y la verdad. Este trabajo explorará las similitudes entre los dos autores también en relación con el retrato Ginevra de, Benci (1475-1478 ca.), de Leonardo da Vinci; una muy especial "sermón pintado" con el fondo del cuadro, siendo un tratamiento filosófico conciso en el que se lee "virtutem forma decorat", fórmula que Ficino evoca en su Convivium.
\end{abstract}

PALABRAS CLAVE: DA VINCI, FICINO, CUSANO, ESTÉTICA, NEOPLATONISMO.

\section{Hand and face The philosophy of expression in Leonardo da Vinci and Marsilio Ficino}

\begin{abstract}
The present paper focuses on Marsilio Ficino and Leonardo da Vinci. Both authors describe the human production of artifacts as transformative principles that affect the world from both a technical and an aesthetic viewpoint. In this light, manus and risus become the strongholds of a typically Renaissance anthropology, according to which the homofaber rises to that godlike status which plays a major role in Nicholas of Cusa's philosophy; while the human hand is the active transformative principle,
\end{abstract}


the smile is the sign of an accomplished work. In sum, these two aspects unveil a process of spiritualization of reality, i.e., the perfect interpenetration of beauty with virtue and truth. This paper will explore the similarities between the two authors also with reference to Leonardo da Vinci's portrait of Ginevra de' Benci (1475-1478 ca.), a very special picture sermon, with the back of the table being a concise philosophical treaty that reads "virtutem forma decorat", a formula which evokes Ficino's Convivium.

KEYWORDS: DA VINCI, FICINO, CUSANUS, AESTHETICS, NEOPLATONISM.

\section{Nicolás de Cusa: el saber técnico y su valor metafórico}

En el período que va desde Leon Battista Alberti y Nicolás de Cusa hasta Marsilio Ficino, Leonardo da Vinci y Alberto Durero, se asiste a la progresiva traducción de una teoría filosófica entrelazada con motivos prevalentemente neo-platónicos a un pensamiento que se hace, sobre la base de los estudios de Eugenio Garin, "un'analisi del fare" (1988: 175). El Quattrocento, de hecho, "è uno dei grandi secoli della tecnica. E per tecnica dobbiamo intendere il giuoco preciso degli strumenti e il loro sfruttamento tanto nella sistemazione dello spazio come nel campo della rappresentazione" (Chastel, 1988: 239).

Una articulación fundamental de esta transformación - de la que emerge, según André Chastel, "un altro tipo di cultura, scaturito largamente dalle curiosità e dalle esperienze familiari alle botteghe, dove si formava l'artifex polytecnes" (ibid.: 258-259)- se encuentra en un texto de Luis Vives, en el que se dice que la verdad "melius agricolae et fabri norunt quam ipsi tanti philosophi" (De disc. III.5: 164).

Además, ya en el Momus de Leon Battista Alberti había un presagio de este cambio de época. Esto se revela cuando el semidiós en el centro de la cuestión afirma que hubiera sido mejor confiar la fabricación del universo a un arquitecto que a un teólogo profesional: el libro de la naturaleza no es solo el fruto de abstracciones conceptuales, sino de determinadas operaciones empíricas gobernadas desde el punto de vista técnico que se apoyan, como en el hacer del artesano, en el numerus, pondus et mensura. Solo los arquitectos serían "capaces de un trabajo tan inmenso" (tanti operis inveniri) como la orbis rerum, una obra artesanal de tal complejidad y precisión que muchos aspectos de la misma parecen "partes de una sustancia divina" (sunt omnia plena deorum) (Momo III: 43). Ya sea que esta posición (en la que hace eco la scientia ignorationis y el sano saber empírico "anti-literario" promovido por el Idiota del Cusano) se traduzca o no en un "manifiesto antifilosófico" -como quisiera Maurice de Gandillac-, lo cierto es que de todos modos ella implica la reivindicación de un saber "consagrado a la conquista del mundo material por medio de la mecánica" (1992: 446).

Por un largo período de pensamiento, más que de un proceso de escolarización del absolutum posse divinum en la facultad humana del libre arbitrio y en la capacidad autónoma de manipulación demiúrgica (técnica, pero también política) de la realidad (Blumenberg, 1996), se asiste en las teorías de la época a una extraña hibridación de motivos metafísicos y técnico-científicos, cuyo vínculo es de recíproca influencia/ derivación metafórica (y su consecuente resignificación). Sobre la base de esta interdependencia figurativa, un tratado sobre mística como el De filiatione Dei traiciona su origen, a nivel de dispositivo técnico, desde una obra como el Della prospettiva de Paolo dal Pozzo Toscanelli, amigo del Cusano desde los tiempos de los estudios en Padua (1417-1423) y de nuevo a su lado en Todi en 1464 junto a "quel Ferdinando Martins portoghese, legato anch'egli, come Toscanelli, all'antefatto del viaggio di Cristoforo Colombo" (Garin, 1998: 125). De hecho, de acuerdo con De filiatione Dei, es evidente cómo la transfusio de la mente humana en el Verbum-speculum infinito de 
la verdad -vale decir, la filiatio Deiperadoptionem, modelada sobre la visión especular frontal, "faccia a faccia" (dal Pozzo Toscanelli, Della prosp., f. 36)- se reubica en el interior de una teoría físico-matemática de la reflexión de los rayos visivos en los "specula recta" (Cusanus, De fil. VI: h IV.65.3): puesto que "alcuna volta lo razo cade sopra lo specchio dirittamente et perpendicolarmente [...], et in quella volta lo razo causa due angoli equali da una parte et da l'altra del specchio"; en modo tal que el rayo incidente coincida en todo y por todo con aquel reflejo, como si "un medesimo razzo si reflette[sse] in sé medesimo" (dal Pozzo Toscanelli, Della prosp., f. 33) -evidente metáfora, en el Cusano, de la unión mística del intellectus con la mens infinita de Dios, fusión visiva para la cual una sola mirada (visio absoluta) al mismo tiempo ve, ejerce el acto de la visión y es objeto de la misma facultad de ver; es aquí, escribe el Cusano, en esta visión de sí, "mens nostra veritatem speculatur" (De fil. VI: h IV.68.4).

Este esqueleto geométrico de la visión directa o frontal de Dios, no casualmente, requiere las mismas condiciones cualitativas que recuerda el Cusano a propósito de la disposición del espejo mental contracto en cuanto rostro en busca del principio intelectual infinito, es decir, que sea nítido, puro, "senza macula di polvere o di altro umore infetto", vale decir, limpio y libre de asperezas así como uniforme (dal Pozzo Toscanelli, Della prosp., ff. 31-32).

La semejanza con el texto del Cusano es notable:

Sintque omnes creaturae specula contractiora et differenter curva, intra quae intellectuales naturae sint viva, clariora atque rectiora specula, ac talia, cum sint viva et intellectualia atque libera, concipito, quod possint se ipsa incurvare, rectificare et mundare ( $D e f i l$. VI: h IV.65.5-9).

No quiero detenerme en la doctrina de Toscanelli del catheto, muy original e innovadora. Diré solamente que ella, combinada con las teorías de la perspectiva del albertino De pictura, es, con una cierta confiabilidad, el origen de la Figura $P$ del Cusano. ${ }^{1}$

Una imagen bellísima, como para sellar esta transición de época, la ofrece Giogione (de Castelfranco) en una pintura enigmática, como para sustentar a cada enfoque inmediato (Hirdt, 2002: 5), en sí mismo empapado de un sorprendente dinamismo: me refiero a I tre magi (también llamado I tre filosofi), obra realizada entre el 1506 y 1508. Esta representación muestra en primer plano la alternancia de tres personajes, desde la derecha hacia el centro de la mesa: un anciano barbudo, quizás Tolomeo; un sabio árabe (mediador, en lo que concierne a los estudios de geometría y de astronomía, entre la Antigüedad clásica y la nueva cultura humanística); y, por último, un joven que intenta observar una caverna equipado con escuadra y compás -y se trata, con mucha probabilidad, de un seguidor de Copérnico. De hecho, pone en la base de su saber "el uso sistemático de la medición y la observación" (de Gandillac, 1992: 488) -y no nos olvidemos que la mente del filósofo, según el Idiota. De mente del Cusano, es idéntica a un "compás vivo" (circinus vivus), capaz de medir todo aquello que existe (De mente 15: h2 V.124.7). Creo que esta exploración triádica representa la evolución de la figura del filósofo según la concepción de Marsilio Ficino y Giovanni

1 Se trata de la duplicación de la figura de lo visible, ya que se refleja en un espejo y se coloca detrás de la misma superficie reflectante del espejo. Esta segunda imagen se transpone a un lugar irreal (tanto como irreales son las imágenes del sueño), de manera tal que contrabalancee la primera pirámide visiva de los "rayos incidentes" con una segunda llamada de los "rayos reflejados", con un ángulo igual y contrario al anterior. En el caso de un reflejo frontal, la pirámide de los rayos incidentes y la pirámide de los rayos reflejados están dispuestas como en la Figura $P$ del Cusano, compenetrándose entre sí, o-como escribe Toscanelli- cayendo en "igualdad con el espejo". Lo que significa, precisamente, que "la catheco cada perpendicolarmente sopra la superficie del specchio [...] et opposita alla cosa visibile faccia a faccia" (dal Pozzo Toscanelli, Della prosp., f. 37). Esta construcción conjetural, con la que "si multiplica quella parte [della realtà] che è opposita al specchio", escribe Toscanelli, da lugar a una segunda imagen que "non è vero corpo ma fantastico, et però non si vede" si no con los ojos de la imaginación (ibid., f. 35). 
Pico, según la cual el pensador debe convertirse en artifex, mientras que el objeto de su especulación asume un rol de la conjetura científica o, incluso, de artificiatum. La fecundidad productiva del artista/artesano hace de estos un segundo Dios (un deus occasionatus, dirá el Cusano): "omnium forma essendi ab illa forma infinita artis aeternae manat sicut artificiatum ab arte" (Sermo XXII: h I.26.6-9). Gracias a estas técnicas, escribe Ficino, el hombre

[t]erram metitur et caelum ac profundas Tartari latebras perscrutatur. Non illi caelum videtur altissimum, ut Mercurii verbis utar, non centrum terrae profundum. Non temporum locorumve intervalla impediunt quin per omnia currat quaecumque sunt in quibuscumque temporibus aut locis. Nullus paries eius aut obtundit aut retundit intuitum. Nulli fines sibi sunt satis. Ubique studet imperare, ubique laudari. Atque ita conatur esse, ut deus, ubique. (Th. Plat. XIV.5).

El éxito de tal evolución es, en efecto, el filósofo-científico experto de las técnicas de medición, que pretende resolver a nivel racional, como objeto de cálculos e hipótesis matemáticas, "le istanze a cui intendevano rispondere e magi e astrologi" de la Antigüedad (Garin, 1988: 173). El saber técnico, que desafía "lingens sylva della natura-materia-mater" (Cacciari, 2016: tab. 10), es aquí la culminación de la filosofía -pero, si se presta atención, nada debe inducirnos a eludir el persistente efecto teológico-metafísico de esta nueva concepción del saber. Las varias experiencias técnicas puestas en el centro del filosofar pueden, entonces, ser entendidas sin duda como modelos heurísticos funcionales al análisis científico real; para ellos, además, esto no se opone a su reutilización transpuesta -podría decirse, en un sentido eminentemente simbólico- dirigida a la recuperación de muchas concepciones filosóficas tradicionales. Aquello que es decididamente nuevo, por lo tanto, es el impulso performativo de este enfoque filosófico para el cual el pensamiento se traduce-incluso solo a nivel de experiencia conjetural- en reelaboración técnico-experimental de la realidad. Ejemplos cusanos de esta tendencia, a la vez técnico-práctica y simbólico-metafísica, son el artesano tallador de cucharas que relucen del Idiota. De mente, la manuductio icónica en el centro del De visione Dei, el ludus trochi sobre el cual se explaya el De possest, el ludus globi de la obra homónima, la referencia al cartógrafo a la que se hace alusión en De theologicis complementis: cada uno de estos exempla tiene un uso tanto científico (por lo tanto racional-empírico) como místico (intelectual), con vínculos explícitos con la teología trinitaria. Estos ejemplos tienen, por lo tanto, el propósito de "lead the reader through a [peculiar] spiritual excercise" en base al cual se desvanece la línea de demarcación entre meditación (también teológica) y juego/experimento mental de orden científico (Moore, 2013: 32). Debido a sus aptitudes prácticas, se traducirán en método experimental verdadero y propio cuando se vuelvan autónomas de su posible uso traslativo-simbólico en otro ámbito (como sucederá más tarde, con Galileo, a propósito de los llamados thought experiments): aquí el juego/experimento perderá cada carácter de manuductio teológico-metafísico, para convertirse en un mero instrumento de la nueva investigación naturalista, "capace di ficcare gli occhi [...] nella materia natura" (Cacciari, 2016: tab. 10), sin ninguna intención de resignificación metafísica.

La secularización, dicho en otros términos, es un proceso que involucrará retrospectivamente a los autores del Renacimiento en primer lugar a nivel semántico: aquello que para ellos era también símbolo de investigación metafísica se convertirá, para los autores posteriores, en instrumento unidireccional de la investigación naturalista. Esta Verveltlichung "of theological presupposition" (Löwith, 1949: 249), podremos también decir, coincide con un proceso de simplificación/especificación lingüística donde la exactitud (la precisión, vista como claridad y distinción) coincide con la inequivocidad del nombre y de la definición, que termina por perder su margen indeterminado 
de significación (o margen de alusión simbólica). ${ }^{2}$ Desde este momento en adelante, la lógica trasuntiva (fundada sobre la cusana transuptio in infinitum) no será más admitida. Además, el verbo transumo, adoptado por el Cusano, significa tomar un término invirtiendo el significado. Transumptio también corresponde a translatio, que es constitutiva del proceso de formación de las metáforas y de los enigmas, en los cuales la atribución del significado -aprovechando la analogía entre planos ontológicos diferentes- puede dar origen a inversiones conceptuales, ${ }^{3}$ más allá de cualquier principio de no-contradicción. Esta posibilidad trasuntiva, escribe Wilhelm Dupré, es para el Cusano entendido "als logischer Inbegriff", es decir como la "Logik der Wahrheit" (Dupré, 1964: 364) misma o la "intellektuale Logik" (más allá del discurso de la ratio discretiva): "Dieser Denk-Weg [...] wird mit Ausdrücken wie 'coincidentia oppositorum', 'symbolice investigare', 'positio lineae infinitae', beziehungsweise [...] 'transumptio sphaerae infinitae ad actualitatem existentiam Dei. Im Unterschied zur rationalen Logik ist sie dem intellectus, dem Denken des Geistes zugeordnet" ibid.: 362-363).

El período de transición examinado está, por lo tanto, marcado por una profunda ambigüedad: lo nuevo es la conciencia del pensamiento de poder formular hipótesis científicas a partir de las cuales es necesario organizar de manera racional el mundo histórico-social; lo que es tradicional, en cambio, se debe al ejemplarismo platónico, que continua determinando la naturaleza participada del espíritu creado, determinándole el carácter de imago et similitudo del principio omnipotente. Entonces, como escribe Rudolf Haubst, cuando el hombre "Indem er sich als Schöpfer seiner Begriffswelt erlebt, gewinnt er zugleich die Möglichkeit, Gott als die sich in den Dingen ideal entfaltende Wahrheit und Wesenheit zu denken" de hecho existentes (1952: 191). El artesano, por su parte, dice que se ocupa de ejercicios que nutren tanto el cuerpo como la mente; "in hac mea arte id, quod volo, symbolice inquiro et mentem depasco, commuto coclearia et corpus reficio" (Cusanus, De sap. II: h² V.55.1).

En el Cusano esta opción dual está representada de modo eminente, y difícilmente pueda ser resuelta unilateralmente:

Coniecturas a mente nostra, uti realis mundus a divina infinita ratione, prodire oportet. Dum enim humana mens, alta dei similitudo, fecunditatem creatricis naturae, ut potest, participat, ex se ipsa, ut imagine omnipotentis formae, in realium entium similitudine rationalia exserit. Coniecturalis itaque mundi humana mens forma exstitit uti realis divina. Quapropter ut absoluta illa divina entitas est omne id quod est in quolibet quod est, ita et mentis humanae unitas est coniecturarum suarum entitas. Deus autem omnia propter se ipsum operatur, ut intellectuale sit principium pariter et finis omnium; ita quidem rationalis mundi explicatio, a nostra complicante mente progrediens, propter ipsam est fabricatricem (De coni. II.17: h III.5.1-10).

Una aclaración adicional. Sobre la referencia al homo cosmographus no se debe olvidar que el Cusano, gran viajero de la época, hacia la mitad del siglo XV diseñó el primer mapa moderno de Alemania (Garin, 1988: 544). Este ejemplo, aunque vuelva clara la nueva sensibilidad científica del pensador, en el plano del filosofar tiene una inmediata confirmación epistemológica: como la búsqueda de la verdad-siendo cada conocimiento racional de orden comparativo- debe partir siempre de un término conocido que sirva de referencia proporcional, así ocurre también en la ciencia cartográfica de la época. También ella se fundaba de hecho en el conocimiento de algunos "punti prefissati" (Singer et al., 2013: 514), a partir de los cuales, de vez en cuando, se podían agregar porciones de realidad cuya noción era adquirida a través

2 Véase mi artículo donde tematizo die Unbestimmtheit des Namen en el ámbito de una Theorie des unbestimmten Bedeutungsspielraums del lenguaje cusano (2019). 
de la investigación empírica (es aquí central el rol de los cinco sentidos, o nuntii visibilium, en su rol de emisarios/exploradores del mundo exterior) (Cusanus, Compendium 13: h XI/3.22.12). Así pues, se podría decir, parafraseando el texto del Cusano, que también el cartógrafo, a través de estos "mensajeros de objetos visibles", pone en relación comparativa elementos de geografía conocidos a partir del mundo todavía desconocido; y así, parte tras parte, se amplía progresivamente la figura huius mundi, llenando los espacios vacíos - al momento todavía desconocidos- del mapa reticular (conjetural) de la órbita terrestre: "studet igitur omni conatu omnes portas habere apertas et continue audire novorum semper nuntiorum relationes et descriptionem suam semper veriorem facere" (ibid.: h XI/3.22.18-20).

También en este caso, la gnoseología del Cusano, al menos a nivel de la facultad senso-racional, no es más que la traducción filosófica de un modelo heurístico válido en el plano de una ciencia empírica: la cartografía. Así como la doctrina mística de la visión, en lo que concierne al esquema geométrico que la soporta, se resuelve en una versión simbólica de los estudios catóptricos, que sirven de base para aplicaciones meta-naturalistas, es decir, de carácter teológico-metafísico.

Omnes autem investigantes in comparatione praesuppositi certi proportionabiliter incertum iudicant; comparativa igitur est omnis inquisitio, medio proportionis utens. Et dum haec, quae inquiruntur, propinqua proportionali reductione praesupposito possunt comparari, facile est apprehensionis iudicium (De docta ign. I.1: h I.5.14-23).

\section{La mano como interpres mundi}

El pasaje de la teoría a la práctica, que es el punto de innovación que se encuentra en el pensamiento del período, está dado por el énfasis puesto sobre la mano: verdadero y propio interpres mundi que permite la articulación de las conjeturas racionales sobre el plano del mundo. Pero este pasaje necesita una aclaración preventiva del término cusano ludus: ciertamente con él se designa el juego tomado en su significado social, pero se trata también de un espectáculo puesto en escena con fines didácticos (dimensiones, estas, bien evidentes en el De visione Dei); además, se trata de una práctica experimental (experimentalis praxis) con la cual se busca una confirmación de la teoría en las condiciones de la experiencia.

La mano, según el Cusano, interpreta los signos mentales que son la base del lenguaje. Mientas el verbo expresa el pensamiento (la imposición del verbum "Impositio igitur vocabuli fit mutu rationis" ad extra) (De mente 15: $\mathrm{h}^{2} \mathrm{~V} .64 .8$ ), la mano -como una extensión, prótesis natural del verbo tomado en su motus- realiza las condiciones de verificación de la doctrina conjetural sobre el plano de la experiencia (confiscando, así, el problema de la definitio a las meras questiones disputatae). El artesano dispone el mundo -su bien delimitada región óntica- en función de una verdad presentida in nuce de la ratio en forma de hipótesis científica: ${ }^{4}$ la mano, que determina las condiciones empíricas hasta que venga a escena un cierto ludus/experimentum, produce un esquematismo pragmático que se coloca entre la conjetura científica, expresada lingüísticamente, y la contraprueba/verificación fáctica de la verdad (de modo que solo al final del proceso es posible hablar de una verdad verificada o veritas formata). Cuando se obtenga la confirmación fáctica de la hipótesis, las consecuencias serán conformes a aquellas estimadas; de esta manera, el praesuppositum teórico será confirmado experimentaliter (De vis. 25: h VI.1.12), o sea, también desde el punto de vista práctico. Gracias al campo de verificación realizado por la mano mediante una cierta configuración lúdica, aquella determinada experiencia -nacida como campo 
de verificación de la hipótesis- debería unirse al mismo tiempo con la conjetura de partida, corroborando esta última. Por lo tanto, si la hipótesis inicial y el juego experimental se corresponden -como en el caso del De visione Dei a propósito de la tabella confiada a los monjes, "figuram cuncta videntis tenens" capaz de esquematizar la visión providencial de Dios (ibid.: h VI.2.12)- la realidad refleja el concepto racional y se obtiene lo que el Cusano llama "adventus formae". Este adventus debe ser entendido como verdad de una cierta experiencia que aflora de una realidad predispuesta/ transfigurada por las sabias operaciones manuales del filósofo-artesano en la forma del ludus/experimentum (en este caso, del ludusiconae): así como, escribe Leonardo en las superficies densas y limpias, gracias al juego/trabajo del artista, se genera finalmente "il lustro" (Cod. Urbinas Lat. 1270, ff. 227v-228r), que alegra la visión gracias a su conformidad con el modelo pensado.

El ejemplo cusano más célebre es aquel del artesano tallador de cucharas, en el lugar central del Idiota. De mente: con los instrumentos a su disposición (martillo, cincel y piedra pómez), este hombre elabora y ahueca la materia de la madera hasta que en la madera misma surja la proporción debida en la cual brille convenientemente la forma de la cuchara (forma coclearitatis convenienter resplendeat) (De mente $15: \mathrm{h}^{2} \mathrm{~V} \cdot 63.7-8$ ). La cuchara, escribe de hecho el Cusano, "extra mentis nostrae ideam aliud non habet exemplar" (ibid.: $\mathrm{h}^{2}$ V.62.8); por lo tanto, también el nombre que lo designa depende de la inventiva humana.

Resplendere es un término que tiene un rol importante en el Sermo CCXLIII; se relaciona con la aparición de la forma como una proporción armoniosa de las partes: "splendor formae [...] super partes materiae proportionatas et terminatas" (Sermo CCXLIII A: h XVII.6.5-7). Solo ab adventu formae la madera recibe el nombre de cuchara: aquí la palabra corresponde a la cosa, entidad cuya esencia es de orden conjetural -al menos hasta que la laboriosidad del hombre no produzca algo que se corresponda en la realidad. "Sic vides formam coclearitatis simplicem et insensibilem in figurali proportione huius ligni quasi in imagine eius resplendere" ( $D e$ mente 15: $\mathrm{h}^{2}$ V.63.8-10). Cito del Sermo XXXIII, donde el vínculo entre nombre y forma es muy explícito: "Advenit igitur omni rei nomen per adventum formae, ut in sigillis cereis dicimus esse illa sigilla regis propter formam regis. Et ex adventu humanitatis ad materiam dicimus individuum hominem; et ita de reliquis" (Sermo XXIII A: h XVII.31.3-5).

El lenguaje, respecto al artificiatum (lo que es producto concretamente de un determinado arte), es una especie de verificación intelectual del éxito de la obra, que se explica en el plano del conocer-como resplendentia de la bella proporción- en la congruencia del fenómeno que debe ser adecuado (en una manera no solo convencional) a la forma del objeto al cual se le ha dado lugar creativamente con el juego de las manos. Pero el advenimiento de la forma, desde el punto de vista del hacer efectivo, se obtiene sobre la base del movimiento de los instrumentos que operan sobre la materia sacando o agregando algo en relación a la forma-verbo tomada intuitivamente de la mente; este objeto (artificiatum), así elaborado, se convierte en la imagen del invenctio conjetural, una especie de espejo ofrecido por la figura asumida, en el ejemplo, por la madera reconfigurada "in figurali proportione" (De mente $15: \mathrm{h}^{2}$ V.64.9). Pero lo mismo podríamos decir de ollas y sartenes, cuya forma es tratada originalmente ex luto:

Non enim in hoc imitor figuram cuiuscumque rei naturalis. Tales enim formae cocleares, scutellares et ollares sola humana arte perficiuntur. Unde ars mea est magis perfectoria quam imitatoria figurarum creatarum et in hoc infinitae arti similior (ibid.: $\mathrm{h}^{2}$ V.62.11-14).

Este proceso de aparición de la formase explica, a su vez, en el movimiento con el cual conferimos el nombre a las cosas: "omne vocabulum eo ipso unitum, quo forma 
materiae advenit"; de modo que "verum sit formam adducere vocabulum", el nombre adecuado para la cosa (De mente 15: $\left.\mathrm{h}^{2} \mathrm{~V} \cdot 59 \cdot 5-9\right)$.

El nombre, aunque no sea preciso y el movimiento de la mente (que lo impone) sea libre (ad beneplacitum) y esté sujeto a un margen de error, es -precisamente en virtud del adeventus formae- "el nombre adecuado" (nomen congruo), es decir, su vocabulum naturale: una suerte de segunda reverberación de la forma sobre el plano de la actividad expresiva de la mente. Concepto y artefacto, en cierto sentido, nacen juntos, y son dos modos de la aparición de la forma ad extra: en el plano de la razón y sobre aquel de la materia. Su unión, como una correspondencia entre teoría y práctica (de la cual emerge el carácter de racionalidad/inteligibilidad del mundo, su concinnitas), está dado por un cierto ludus o experimentalis praxis, como una extensión del lenguaje a través de los juegos operativos de la mano: el movimiento del nombre y el movimiento de los instrumentos técnicos hacen que surja el nombre/forma de un cierto artefacto. Y esto en nombre de la experiencia de verdad a la que la actividad del lenguaje y de la mano corresponde un momento de pasividad intuitiva con respecto al darse revelate de la forma (el adventus en el que a un momento expresivo de humana operatividad se agrega un momento revelador, de tipo trascendente). 5 "Et quamvis lignum recipiat nomen ab adventu formae, ut orta proportione, in qua coclearitas resplendet, 'coclear' nominetur, ut sic nomen sit formae unitum" (De mente 15: $\mathrm{h}^{2} \mathrm{~V} .64 \cdot 1-3$ ).

Existe, por lo tanto, un cierto isomorfismo dinámico entre producción de los "instrumenta pro arte mechanica" (Sermo X: h XVI.28.36), concepción de los conceptos e imposición de los nombres. Así pues, el hacer del artesano se convierte en esquema de toda la actividad del espíritu creado. En sus operaciones se manifestaría de hecho la "explicação da sua força intelectual [de la mens], imagem da explicação da força criadora divina" (André, 1999: 16). En este sentido, la esencia del homo artifex, sobre la base del carácter dinámico ofrecido por el motivo cusaniano-ficiano del hombre ad Dei immortalem imaginem (Ficino, De raptu Pauli, Op. Th. XXVIII: 965), coincidiría con el ser un "intérprete de símbolos e criador de símbolos", así como un "produtor de cultura" material (André, 1999: 23).

\section{La obra lograda: Ficino, Leonardo y el risus como splendor formae}

Como para el Cusano la mano es intérprete de aquellos signos mentales que se expresan en el lenguaje, para Leonardo, nuestro juicio es aquello que mueve la mano: "Questo accade, che il giudizio nostro è quello che muove la mano alle creazioni de" lineamenti di esse figure per diversi aspetti insino a tanto ch'esso si satisfaccia" (Trat. della pitt. III.487). Esta concepción encuentra una confirmación precisa en la doctrina de la pintura; $y$, en efecto,

$$
\begin{aligned}
& \text { ciò che è nell’universo per essenza, presenza o immaginazione, esso [il pittore] lo ha prima } \\
& \text { nella mente, e poi nelle mani, e quelle sono di tanta eccellenza, che in pari tempo generano } \\
& \text { una proporzionata armonia in un solo sguardo qual fanno le cose (Trat. della pitt. I.9). }
\end{aligned}
$$

Las manos, por lo tanto, también para Leonardo son los medios para la realización de la proporción (tomada por la razón) de la realidad; y es gracias a su movimiento laborioso que nace la belleza sensible -como una suerte de suceso de verdad, bajo la forma de resplendentia, por la cual los "accidentes mentales"- a la par de las conjeturas cusanas -toman forma en el mundo de la materia, compenetrándola en sentido espiritual. En todo esto, evidentemente, Leonardo parece estar guiado por la 
afirmación de Ficino según la cual la belleza es "risplende nel corpo per lo influsso della sua Idea" (Convivium V.6: 78).

Pero no es este el centro de la teoría de la belleza de Leonardo. Más bien, se encuentra en la particular concepción del visus como expresión de esa armonía espiritual en la que consiste la formositas. La dementus formae, para Leonardo, tiene lugar entonces en el plano de la relación artística del rostro; en él, de hecho se concentra la vida espiritual del individuo (a su vez centro indiscutido del arte del retrato, vértice de la ciencia pictórica): "Gli accidenti mentali muovono il volto dell'uomo in diversi modi" (Trat. della pitt.III.282); alguno de ellos hacen reír, otros llorar, otros regocijarse, otro pensar y especular, etc. Ahora, "questi accidenti debbono accompagnare le mani con il volto, e così la persona" (ibid.).

Sobre esta base, muchas páginas del Trattato della pittura resultan ser, in nuce, un tratado de fisonomía, en el que el rostro se convierte en el punto de intersección entre piramis lucis (mundo del espíritu) y piramis tenebris (mundo ensombrecido de la materia):

Vero è che i segni de' volti mostrano in parte la natura degli uomini, i loro vizi e complessioni; ma nel volto i segni che separano le guancie dai labbri della bocca, e le nari del naso e le casse degli occhi sono evidenti, se sono uomini allegri e spesso ridenti; e quelli che poco li segnano sono uomini operatori della cogitazione; e quelli che hanno le parti del viso di gran rilievo e profondità sono uomini bestiali ed iracondi, con poca ragione; e quelli che hanno le linee interposte infra le ciglia forte evidenti sono iracondi, e quelli che hanno le linee trasversali della fronte forte lineate sono uomini copiosi di lamentazioni occulte e palesi (III.288).

El presupuesto estético-metafísico de estas doctrinas artísticas de Leonardo se relaciona con la metafísica de la luz de Ficino; ella está centrada en el splendordivinae bonitatis, en el cual hace eco también la tesis cusana del adventum formae. En una carta al jurisconsulto Bernardo Bembo, Ficino afirma que la luz es la perfección de la forma o, literalmente, "formositas consistit in luce" (De luce Dei, Op. Th. IX: 274), la cual se manifiesta sobre la superficie de las cosas como un leve sorriso-risus que parece expandirse sobre el rostro del mundo con un impulso vibrante que aporta proporción y armonía (que conduce de nuevo a la unidad las partes conmensuradas de materia, diría el Cusano): "risus caeli ex numinum gaudio proficiscens, id est lumen, omnia fovent atque delectat", se lee en los Opuscula theologica (Quid sit lumen, VII: 375). A tal sonrisa (que es un resplandor de la luz interior, divina, en el mundo material, como bien había entendido Leonardo da Vinci), la mente corresponde con el impulso amoroso: antiquísimo Dios es amor, señala Ficino, ya que es la misma propagación del soplo de Dios en el mundo, que quiere reconducir la realidad a si misma (cerrando el círculo de explicación de lo real con la coincidencia del origen y destino último), para convertir finalmente las mentes al principio mismo.

La filosofía de Ficino es, a veces, un verdadero himno al amor; y el amor, siempre según Ficino, es indiscutiblemente el verdadero autor del arte. Escribe Chastel, "c'est l'amour qui sculpte, l'amour qui peint, et cette statue, cette image cachée dans le coeur, deviennent vite si précieuses qu'elles se substituent à leêtre aimé [así reconfigurado], car c'est à leur niveau que s'opère le passage du sensible à l, 'idée'" (1975: 125). Igualmente significativo es el hecho que en el Commentarium in Convivium Platonis de amore Ficino afirme que el amor es el maestro y señor de todas las artes, artes que comienzan a unificar la multiplicidad (creando armonía) en cuanto inspiradas por 
las Musas: "poesis a divino furore, furor a Musis, Musae vero a Jove proficiscuntur" (Epist. I: 614). ${ }^{6}$

Pero hay una pintura de Leonardo en particular que debemos investigar aquí, en la cual la reanudación de motivos cusanos por parte de Ficino encuentra casi su emblema en un tiempo icónico y doctrinal: se trata del retrato de Ginevra de Benci, realizado entre 1475 y 1478 (National Gallery of Art, Washington D.C.). La imagen, en efecto, es doble; sobre la parte posterior de la mesa se reporta un lema rodeado por una suntuosa guirnalda de enebro (iuniperus, que alude al nombre Ginevra), una frase que suena así: virtutem forma decorat. Esta expresión, bien entendida, tiene una clara comparación con el ya citado Sermo CCXLIII del Cusano, en el cual la belleza -tras las huellas del Commentarium al De divinis nominibus de Alberto Magno (Cod. Cus. 96)- es definida como el resplandor de la forma sobre las partes proporcionales de la materia (Sermo CCXL: 32): aquella "relazione armoniosa e gradevole fra le singole parti" de una realidad dada, escribe Kristeller (1942: 328), tal que difunde sobre ella el leve risus sobre el cual Ficino y Leonardo se extendieron. Para Leonardo en particular, la forma o la belleza, desde el punto de vista moral, es aquello que decora la virtud y el valor, que serían dotes intrínsecas y del todo discretas en el plano fenoménico si no fueran reveladas por la elegancia y por la belleza de la figura realizada por el arte (Zöllner, 2010: 38).

En este sentido, también para Ficino la armonía del cosmos, o el orden natural establecido por Dios, no es otra cosa que la imago divinae bonitatis (Th. Plat. II.13). Por lo tanto, dado este orden, "in necessariis rebus natura non deficit, supervacuis non abundat" (Th. Plat.VII.2). A través de la proporción advertida como agradable, el objeto externo (aprobado, por así decir, en el juicio estético) se refiere en última instancia "al concetto interiore e attraverso questo allidea divina dell'uguaglianza" (Kristeller, 2005: 330).

Pero en el Convivium de Ficino se encuentra una verdadera y propia explicación de la fórmula leonardesca, como para pensar una recuperación icónica, en el retrato realizado por Leonardo, de un motivo puramente neoplatónico de matriz cusana-ficiniana:

La virtù dello animo mostra di fuori un certo ornamento nelle parole, ne' gesti, e nelle opere onestissimo [...]. In tutte queste cose la perfezione di dentro produce la perfezione di fuori: e quella chiamiamo Bontà, questa Bellezza. Per la qual cosa vogliamo la Bellezza essere fiore di Bontà. E per gli allettamenti di questo fiore, quasi come per una certa esca, la Bontà ch’è dentro nascosa, alletta i circustanti (V.1: 67-68).

Para Leonardo, la forma artística, "la speziosa figura del corpo" (Convivium V.2: 70), como resplendentia de la esencia armoniosa de lo real, dicho en otros términos, manifiesta y hace inteligible la idea como una razón intrínseca de la disposición armoniosa de lo real, así como lo bello "una manifestazione di segrete leggi della natura" (Cassirer, 1950: 251). Representar, delinear la forma no es posible, por lo tanto, sin el ejercicio de la teoría, de aquella sutilísima especulación pictórica como visione interna, metafísica, del mundo de las cosas; de modo que el rostro retratado, obra de un gran trabajo artístico, no es más que metáfora de la fuerza espiritual que atraviesa la naturaleza: esta última emerge -como adventus formae-como aquella leve sonrisa que atraviesa la realidad.

Ver internamente la realidad, tomar su inmanente configuración que se reverbera en una imagen bien proporcionada, significa literalmente intuirla: intus legere, casi un "tatto della realtà per di dentro" (Bongioanni, 1935: 19), que captura la verdad 
gracias a su fuerza ostensiva que aparece -como el brillar de la forma- en la belleza. Se podría decir que para Leonardo no hay verdad que no tenga la fuerza de aparecer, manifestándose como speciosa forma-y en esto está el valor filosófico de la pintura, capaz de penetrar en la estructura eidética de lo creado de otra manera que la palabra (Solmi, 1905: 39). Así, como escribe Ernst Cassirer,

l'arte non è mai soltanto una creazione della fantasia soggettiva, ma è, e rimane, un vero ed indispensabile organo per l'apprensione dello stesso reale. Il valore di verità immanente a questa non è inferiore a quello della scienza [...]. Leonardo, anche come investigatore, non si diparte da questa visibilità e concepibilità della forma (1950: 248).

Por otro lado, como Leonardo pudo fácilmente encontrar en Ficino, la bella figura, emergiendo en la materia en la leve sonrisa -junto al sonido armonioso y, obviamente, en la verdad racional- pertenece más al alma que al cuerpo: "Razón, Vista y Oído", facultades que corresponden a la ciencia (o virtud), figura y voz, pueden ser así representadas como las tres Gracias que acompañan al alma del artista, o sea Esplendor, Vigor y Alegría (definiciones de las Gracias, esta última, que también encontramos en Giovanni Pico).?

Orfeo chiama splendore quella grazia e bellezza dell'animo, la quale nella chiarezza delle scienze e de' costumi risplende; e chiama viridità cioè verdezza, la suavità della figura e del colore: perché questa massime nella verde gioventù fiorisce: e chiama letizia, quel sincero, utile e continovo diletto, che ci porge la Musica (Ficino, Convivium V.2: 71).

Solo una verdad bella, como había comprendido Ficino, supera la razón discursiva y cada pretensión de posesión material; solo en tanto belleza, "immagine dell’ornamento divino" (ibid. II.7: 41), alimenta nuestro deseo de verdad, aquel furor amoroso de perfección que se dirige a tomarla con las tres facultades espirituales Mente, Vista y Oído. Estas facultades corresponden a las tres musas Esplendor, Vigor (o Verdor) y Alegría abundante, las cuales, como en la Primavera botticelliana, rodean bailando la "fiore di Bontà" (Convivium V.1: 68) -"Venere che le Grazie la fioriscono, dinotando la Primavera", según el Vasari (Le vite: I.475).

El risus que anuncia la redescubierta armonía del cosmos es la bondad que brilla bajo el manto de la belleza, emergencia tal que deja la realidad traslúcida, legible en su fundamento noético. Esto es también la tarea que se pone Botticelli, cuyo fundamento pedagógico fue sabiamente orientado por Ficino. Cito de una carta dirigida a Lorenzo de Médici y a Bernardo Bembo, en la que la doctrina filosófica de Ficino se traduce casi en imagen (en un modo muy próximo a las teorías leonardescas del Trattato della pittura):

Multa Philosophi disputant, Oratores declamant, poetae canunt, quibus homines exhotentur ad verum virtutis amorem. [...] Sed puto virtutem ipsa, (si quando producatur in medium) multo facilius meliusque quam verba hominum ad se capessendam cunctos adhortaturam. Frustra puellam adolescentis auribus laudas verbisque describis, quo stimulos illi amoris incurias [...] Monstra, (si potes) formosam digito, nihil amplius hic tibi opus est verbis. Dici enim non potest quando facilius vehementiusque pulchritudo ipsa, quam verbam provocet ad amandum. Ergo si mirabilem virtutis ipsius speciem in conspectum hominum proferamus, haud opus erit suasionibus nostris ulterius, ipsamet citius, quam cogitari possit persuadebit (Epist. V: I.807).

7 "I poeti affermano che Venere ha come compagne e sue fanciulle le Grazie, i cui nomi nella lingua volgare sono Verdezza, Gioia e Splendore. Queste tre Grazie non sono che le tre proprietà tipiche della Bellezza Ideale” (Gombrich, 1972: 83). 
Para concluir. El adventus formae (gracias al poder de transfiguración espiritual del risus) es un evento que es difícil de explicar. El Cusano y Ficino han tomado la profunda raíz teológica de este silencio cargado de doctrina. En la Theologia platonica, libro $\mathrm{X}$, Ficino afirma que Dios, tal como el artista hace con la materia, tiene como objetivo trazar la forma, es decir, la imagen de la perfección de su rostro, de la naturaleza creada en la cual obra: "quam ut eam [materiam] extollat ad sui vultus imaginem". El hombre, a imitación de Dios, hace lo mismo en el mundo del arte y de la técnica; es entonces necesario que, gracias al uso sabio de las manos, en algún lugar de la materia, estimulada por mucho tiempo, brille en el modo más claro la faciem arteficis. En estas obras, de hecho, el alma humana tiene una forma de expresarse y manifestarse al exterior tal como "vultus hominis intuentis in speculum seipsum figurat in speculo" (Th. Plat. X.4). Ahora bien, en esta imagen del rostro humano se refleja también el principio divino, modelo de toda creación humana: imago Dei e imago hominis, puestas una frente a la otra, dan lugar a aquella visión frontal de la cual partimos, visio caracterizada por una doble transfusión de las miradas (recíproca y complementaria) bien representada de la Figura $P$. Y se trata de un único resplandor que es el adventus formae: advenimiento realizado por manos humanas, pero solo en tanto inspiradas en un arquetipo eterno de belleza, que se apodera transfigurando los datos de la naturaleza. Su sonrisa que trae orden es evidente en toda creación humana, en tanto bella y proporcionada; mientras el hombre, en el apogeo de su libertad de expresión, se descubre co-creador. Ser artista, para Leonardo, equivale a ser un conditor en el centro de un mundo de conjeturas artísticas hechas de lineamientos, perspectivas y colores. Pero en cada rostro realizado, la sonrisa traiciona el origen de toda belleza de una forma única (la forma formarum): no tiene igual, y puede ser solo presagiada como un ideal de perfección. 


\section{Bibliografía}

\section{Fuentes}

\section{Manuscritos}

» Leonardo da Vinci. Libro di pintura. Vaticano, Bibl. Ap. Vat., Codex Urbinas Latinus 1270.

\section{Ediciones}

" Giorgio Vasari (2010). Le vite de' più eccellenti architetti, pittori, et scultori italiani, da Cimabue insino a' nostri tempi. Nell'edizione per i tipi di L. Torrentino (Florencia, 1550). Ed. Bellosi, L. y Rossi, A. Turín: Einaudi.

» Juan Luis Vives (1764). De disciplinis. III: De causis corruptarum artium. Napoles: Typographia Simoniana.

»Leon Battista Alberti (1992). Momo o del principe. Ed. lat. y trad. it. Consolo, R. Génova: Costa \& Nolan.

"Leonardo da Vinci (2002). Trattato della pittura., en Scritti. Tutte le opere: Trattato della pittura, Scritti letterari, Scritti scientifici. Ed. Recupero, J. Milán: Rusconi.

" Marsilio Ficino (1576). Epistolarium, en Opera omnia I, Ed. Henricus Petri. París.

» Marsilio Ficino (2003). Sopra lo amore ovvero Convito di Platone. Ed. Rensi, G. Milán: SE. (Testi e documenti 31).

» Marsilio Ficino (2007). Théologie platonicienne de l'immortalité des âmes. Suivi des Opuscula theologica. Ed. lat. y trad. fr. Marcel, R. París: Les Belles Lettres.

"Nicolai de Cusa (1932 ss.). Nicolai de Cusa Opera Omnia. Eds. Hoffmann, E., Bormann, K., Klibansky, R., \& der Wissenschaften, H. A. Leipzig-Hamburgo: Meiner.

» Paolo dal Pozzo Toscanelli (1991). Della prospettiva. Ed. Parronchi, A. (rip. anast. del Cod. Riccardiano n. 2110). Milán: Polifilo.

\section{Bibliografía complementaria}

"André, J. M. (1999). "O Homem como Microcosmo. Da concepção dinâmica do homem em Nicolau de Cusa à inflexão espiritualista da anthropologia de Ficino", Philosophica 14, 7-30.

" Blumenberg, H. (1996). Die Legitimitat der Neuzeit. Frankfurt a.M.: Suhrkamp.

"Bongioanni, D. (1935). Leonardo pensatore. Saggio sulla posizione filosofica di Leonardo da Vinci. Plasencia: Soc. Tipografica Ed. Porta.

" Cacciari, M. (2016). "Ripensare l'Umanesimo”. En Egbi, R. (ed.). Umanisti italiani. Pensiero e destino. Turín: Einaudi.

" Cassirer, E. (1950). Individuo e cosmo nella filosofia del Rinascimento. Trad. it. Federici, F. Florencia: La Nuova Italia.

»Chastel, A. (1975). Marsile Ficin et l'art. París: Droz. 
" Chastel, A. (1988). "L'artista". En Garin, E. (ed.). L'uomo del Rinascimento. RomaBari: Laterza, 239-269.

»Cuozzo, G. (2019). "Das Paradox als sprachliche Möglichkeit in der philosophischen Erkenntnistheorie. Von Cusanus zur Postmoderne", MFCG 35, en prensa.

" de Gandillac, M. (1992). Genèse de la modernité. De la "Cité de Dieu" à la "Nouvelle Atlantide". París: eds. du Cerf.

» Dupré, W. (1964). “Die Idee einer neuen Logik bei Nikolaus von Cues", MFCG 4, 357-374.

» Forti, F. (1967). "La 'transumptio' nei dettatori bolognesi e in Dante". En Dante e Bologna nei tempi di Dante. Bolonia: Facoltà di Lettere e Filosofia dell'Università di Bologna.

» Garin, E. (1988). “Il filosofo e il mago". En L'uomo del Rinascimento. Roma-Bari: Laterza, 169-202.

» Garin, E. (1998). La cultura del Rinascimento. Milán: Il Saggiatore.

» Gombrich, E. H. (1972). Immagini simboliche. Studi sull'arte del Rinascimento. Turín: Einaudi.

" Haubst, R. (1952). Das Bild des Einen und Dreieinen Gottes in der Welt nach Nikolaus von Kues. Tréveris: Paulinus. (Trierer Theologische Studien Bd. 4).

» Hirdt, W. (2002). Bildwelt und Weltbild. "Die Drei Philosophen" Giorgiones. Tubinga: A. Franke.

» Kristeller, P. O. (2005). Il pensiero filosofico di Marsilio Ficino. Florencia: Le Lettere.

" Löwith, K. (1949). Meaning in History. The Theological Implications of the Philosophy of History. Chicago: University of Chicago Press.

» Malagoli, L. (1949). Linguaggio e poesia nella Divina Commedia. Génova: Briano.

» Marzot, G. (1956). Il linguaggio biblico nella Divina Commedia. Pisa: Nistri-Lischi.

» Moore, M. E. (2013). Nicholas of Cusa and the Kairos of Modernity. Nueva York: Punctum.

» Oliviero, F. (1936). The Representation of the Image in Dante. Turín: S. Lattes e C.

» Pareyson, L. (1971). Verità e interpretazione. Milán: Mursia.

» Singer, C., Holmyard, E. J., Hall, A. R. y Williams, T. I. (eds.) (2013). Storia della tecnologia. (v. 3/2: Il Rinascimento e l'incontro di scienza e tecnica). Turín: Bollati Boringhieri.

»Solmi, E. (1905). Nuovi studi sulla filosofia naturale di Leonardo da Vinci. Il metodo sperimentale. L'astronomia. La teoria della visione. Mantua: G. Mondovì.

»Zöllner, F. (2010). Leonardo da Vinci. Sämtliche Gemälde und Zeichnungen. Colonia: Taschen. 